\title{
Stability Analysis and Controller Design for Dynamic Fuzzy Systems Based on a New Fuzzy Inference Approach $^{1}$
}

\author{
Elmar Schäfers \\ Universität Karlsruhe, Institut für Regelungs- und \\ Steuerungssysteme \\ Kaiserstr. 12, D-76131 Karlsruhe, Germany \\ E-mail: schaefer@irs.etec.uni-karlsruhe.de
}

\begin{abstract}
Qualitative modeling of technical processes may be accomplished by dynamic fuzzy systems. A new inference method with interpolating rules is proposed as an essential basis for the analysis of this class of systems. Using this approach, the system output is dependent on both an interpolating rule derived from the fuzzy input and the fuzzy input itself. A simple example shows the typical behavior of such dynamic fuzzy systems and leads to a stability definition. Furthermore, it is shown how a fuzzy controller is designed using a set of fuzzy rules describing the process behavior. The design method is applied to a continous stirred tank reactor.
\end{abstract}

\section{Keywords:}

Dynamic fuzzy systems, fuzzy controller design, inference with interpolating rules, stability analysis of dynamic fuzzy systems

\section{Introduction}

Most control engineering applications of fuzzy logic are based on a set of rules with fuzzy premises and fuzzy conclusions. To describe complex processes qualitatively, a fuzzy output dependent on fuzzy input variables has to be calculated. There exist various reasoning methods $[1,6]$ with particular interpretation of the fuzzy rules.

The shape of the membership function of the fuzzy output calculated with the commonly used reasoning

\footnotetext{
${ }^{1}$ This work was supported by the Deutsche Forschungsgemeinschaft (DFG) under contract No. Kr 949/5-1.
}

\author{
Volker Krebs \\ Universität Karlsruhe, Institut für Regelungs- und \\ Steuerungssysteme \\ Kaiserstr. 12, D-76131 Karlsruhe, Germany \\ E-mail: krebs@irs.etec.uni-karlsruhe.de
}

methods (e.g. "max-min-inference" or "max-prodinference") is generally different from the shape of the membership functions of the premises and conclusions. If, for instance, all membership functions of the conclusions are fuzzy numbers, the membership function of the fuzzy output is generally not a fuzzy number.

An inference method is expected to evaluate a set of fuzzy rules corresponding to the human way of approximate reasoning. Due to the fact that human beings are able to process only such fuzzy sets that might be properly adjoined to linguistic values, only this kind of fuzzy sets are appropriate inputs of fuzzy systems. Since the membership functions of the premises and conclusions are user-defined to mark linguistic values e.g. as fuzzy numbers, they might be viewed as understandable fuzzy sets. Considering in particular dynamic fuzzy systems that feedback the fuzzy output, it has to be guaranteed that the inference maps understandable fuzzy inputs onto an understandable fuzzy output.

In the following, a new fuzzy inference method called "inference with interpolating rules" is presented that guarantees an output of a fuzzy system belonging to the same class of fuzzy sets as the fuzzy inputs. In this contribution, triangular fuzzy numbers are chosen as understandable fuzzy sets. In the third chapter, it is shown that dynamic fuzzy systems feedbacking the fuzzy output produce suitable results with this new inference method. Furthermore, a new stability definition for dynamic fuzzy systems and an approach for stability analysis are presented. The fourth chapter focuses on a new design strategy for fuzzy controllers and the final chapter presents an application of the new method. With this new approach, it is possible to develop a fuzzy controller only from a linguistic description of the process behavior. 


\section{Inference with interpolating rules}

The rules of the fuzzy rule set are considered as "fundamental rules". Together with the fuzzy inputs the fundamental rules allow to determine interpolating rules. An interpolation between premises and conclusions of the fuzzy rule set yields the interpolating premise and the interpolating conclusion of the interpolating rule. Finally, the fuzzy output results from the interpolating rule considering the fuzzy input.

\subsection{Assumptions}

The membership functions of all premises, conclusions, and inputs have to belong to a class of functions that can be described with a finite number of parameters. In this contribution triangular fuzzy numbers $\left\langle v_{c} ; v_{l}, v_{r}\right\rangle$ are used that are clearly defined with their center $v_{c}$ and the coordinates $v_{1}$ and $v_{r}$ of their left and right foot, respectively. An interpolation is only possible if the coordinates of the left and right foot as well as the center of the adjacent premise membership functions are different. Furthermore, it is presupposed that the centers $\operatorname{con}_{\mathrm{c}}^{\mathrm{i}}$ of all conclusions with the coordinates $\left\langle\operatorname{con}_{\mathrm{c}}^{\mathrm{i}} ; \operatorname{con}_{1}^{\mathrm{i}}, \operatorname{con}_{\mathrm{r}}^{\mathrm{i}}\right\rangle$ are different from each other and that $\operatorname{con}_{1}^{\mathrm{i}} \leq \operatorname{con}_{1}^{\mathrm{j}}$ as well as $\operatorname{con}_{\mathrm{r}}^{\mathrm{i}} \leq \operatorname{con}_{\mathrm{r}}^{\mathrm{j}}$ follows from $\operatorname{con}_{\mathrm{c}}^{\mathrm{i}}<\operatorname{con}_{\mathrm{c}}^{\mathrm{j}}$ with $\mathrm{i} \neq \mathrm{j}$. These last two conditions represent no restriction to the method and are only introduced to avoid the consideration of some special cases affecting the transparency of this contribution.

\subsection{Determination and evaluation of an interpolating rule}

The following two fuzzy rules with one input and one output are used to explain the new inference mechanism, the extension to multiple input multiple output systems is straightforward:

IF "Input is small" THEN "Output is large"
IF "Input is large" THEN "Output is small"

The membership functions of the linguistic values "small" and "large" of the input are triangular fuzzy numbers $A<\mathrm{is}_{\mathrm{c}} ; \mathrm{is}_{\mathrm{l}}, \mathrm{is}_{\mathrm{r}}>$ and $B<\mathrm{il}_{\mathrm{c}} ; \mathrm{il}_{\mathrm{l}}, \mathrm{il}_{\mathrm{r}}>$, the linguistic values "small" and "large" of the output are the triangular fuzzy numbers $C<\mathrm{os}_{\mathrm{c}} ; \mathrm{os}_{1}, \mathrm{os}_{\mathrm{r}}>$ and $D$ $<\mathrm{ol}_{\mathrm{c}} ; \mathrm{ol}_{1}, \mathrm{ol}_{\mathrm{r}}>$, respectively. Considering a fuzzy input Inp $<\operatorname{inp}_{\mathrm{c}} ; \operatorname{inp}_{\mathrm{l}}, \operatorname{inp}_{\mathrm{r}}>$, the parameters of the interpolating premise $I P<\mathrm{p}_{\mathrm{c}} ; \mathrm{p}_{1}, \mathrm{p}_{\mathrm{r}}>$ and the interpolating conclusion $I C<\mathrm{c}_{\mathrm{c}} ; \mathrm{c}_{1}, \mathrm{c}_{\mathrm{r}}>$ of the rule set (1) have to be determined. A measure for the distance between two fuzzy numbers

$$
\mathrm{d}\left(\mathrm{A}_{1}, \mathrm{~A}_{2}\right)=\text { center }\left(\mathrm{A}_{1}\right)-\operatorname{center}\left(\mathrm{A}_{2}\right)
$$

and related distances of $\operatorname{Inp}$ to $A$ and $B$

$$
\begin{aligned}
& \tilde{a}=\frac{d(\operatorname{Inp}, A)}{d(B, A)}=\frac{i n p_{c}-i s_{c}}{i_{c}-i s_{c}}, \\
& \tilde{b}=\frac{d(\operatorname{Inp}, B)}{d(A, B)}=\frac{i n p_{c}-i l_{c}}{i s_{c}-i l_{c}}=1-\tilde{a} .
\end{aligned}
$$

are introduced. The proportion of the two rules IF "Input is small" THEN "Output is large" and IF "Input is large" THEN "Output is small" to the interpolating rule IF "Input is $I P$ " THEN "Output is $I C$ " correspond to the related distances $\tilde{\mathrm{a}}$ and $\tilde{\mathrm{b}}=1-\tilde{\mathrm{a}}$. Thus, the parameters of $I P$ and $I C$ are

$$
\begin{aligned}
& \mathrm{p}_{\mathrm{c}}=\tilde{\mathrm{a}} \cdot \mathrm{il_{ \textrm {c } }}+(1-\tilde{\mathrm{a}}) \cdot \mathrm{is}_{\mathrm{c}}, \quad \mathrm{p}_{1}=\tilde{\mathrm{a}} \cdot \mathrm{il_{1 }}+(1-\tilde{\mathrm{a}}) \cdot \mathrm{is}_{1}, \\
& \mathrm{p}_{\mathrm{r}}=\tilde{\mathrm{a}} \cdot \mathrm{i} \mathrm{l}_{\mathrm{r}}+(1-\tilde{\mathrm{a}}) \cdot \mathrm{is}_{\mathrm{r}}
\end{aligned}
$$

and

$$
\begin{aligned}
& \mathrm{c}_{\mathrm{c}}=\tilde{\mathrm{a}} \cdot \mathrm{os}_{\mathrm{c}}+(1-\tilde{\mathrm{a}}) \cdot \mathrm{ol}_{\mathrm{c}}, \quad \mathrm{c}_{1}=\tilde{\mathrm{a}} \cdot \mathrm{os}_{1}+(1-\tilde{\mathrm{a}}) \cdot \mathrm{ol}_{1}, \\
& \mathrm{c}_{\mathrm{r}}=\tilde{\mathrm{a}} \cdot \mathrm{os}_{\mathrm{r}}+(1-\tilde{\mathrm{a}}) \cdot \mathrm{ol}_{\mathrm{r}} .
\end{aligned}
$$

The center of the input inp $\mathrm{c}_{\mathrm{c}}$ is always equivalent to the center of the interpolating premise IP. If INP is included in $I P, I N P$ is a fuzzy set with the same center and at least the same specificity (defined e.g. in [6]) as $I P$. Therefore, it is straightforward to choose the output Out $<$ out $_{\mathrm{c}}$; out $_{1}$, out $_{\mathrm{r}}>$ equivalent to IC (fig. 1).
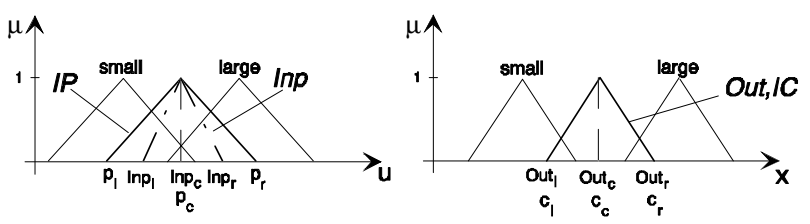

Figure 1. Input included in the interpolating premise

If the fuzzy input Inp is not included in the interpolating premise (see e.g. fig. 2), one or both feet are outside of the support of $I P$. Hence, the parameter of an outlying output foot is appropriately calculated by interpolation between the feet of the membership functions of the linguistic values "small" and "large" of the output as follows: 
out $_{1}=\tilde{\mathrm{a}}_{1} \cdot \mathrm{os}_{1}+\left(1-\tilde{\mathrm{a}}_{1}\right) \cdot \mathrm{ol}_{1} \quad$ out $_{\mathrm{r}}=\tilde{\mathrm{a}}_{\mathrm{r}} \cdot \mathrm{os}_{\mathrm{r}}+\left(1-\tilde{\mathrm{a}}_{\mathrm{r}}\right) \cdot \mathrm{ol}_{\mathrm{r}}$

$\tilde{a}_{1}=\left\{\begin{array}{lc}\operatorname{inp}_{\mathrm{r}}>\mathrm{p}_{\mathrm{r}}: & \frac{\mathrm{inp}_{\mathrm{r}}-\mathrm{is}_{\mathrm{r}}}{\mathrm{il}_{\mathrm{r}}-\mathrm{is}_{\mathrm{r}}} \\ \operatorname{inp}_{\mathrm{r}} \leq \mathrm{p}_{\mathrm{r}}: & \tilde{\mathrm{a}}\end{array} \tilde{\mathrm{a}}_{\mathrm{r}}=\left\{\begin{array}{lc}\operatorname{inp}_{1}<\mathrm{p}_{1}: & \frac{\mathrm{inp}_{1}-\mathrm{is}_{1}}{\mathrm{il}_{1}-\mathrm{is}_{1}} \\ \operatorname{inp}_{1} \geq \mathrm{p}_{1}: & \tilde{\mathrm{a}}\end{array}\right.\right.$.

Figure 2 depicts the output Out in case of an input Inp with only its right foot inp $\mathrm{r}_{\mathrm{r}}$ outside of the support of IP. Consequently, the left foot out ${ }_{1}$ of the output membership function is outside the support of $I C$, whereas the right foot out $\mathrm{r}_{\mathrm{r}}$ of the output is equivalent to the right foot $\mathrm{c}_{\mathrm{r}}$ of $I C$. Obviously, moving the right foot of Inp causes a relocation of the left foot of Out due to the cross-over of the rules (1).
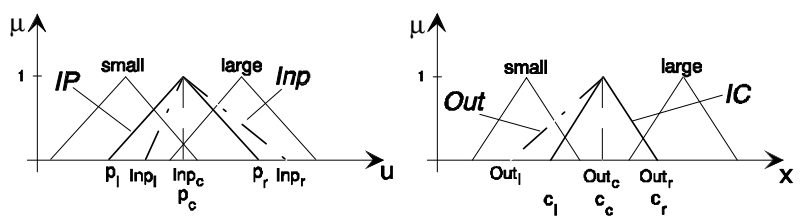

Figure 2. Input not included in interpolating premise

Two adjacent membership functions of the input define an interpolation domain. In order to avoid that the transition of the input from one interpolation domain to another is not continuous, fuzzy interpolation domains have to be established by allocating continuous membership functions to each interpolation domain. To sum up this section, the presented inference method guarantees a continuous mapping of understandable fuzzy inputs onto an understandable fuzzy output.

\section{Stability Analysis of Fuzzy Systems}

In this section, dynamic fuzzy systems feedbacking the fuzzy output are considered. As an example, figure 3 depicts a first order dynamic fuzzy system.

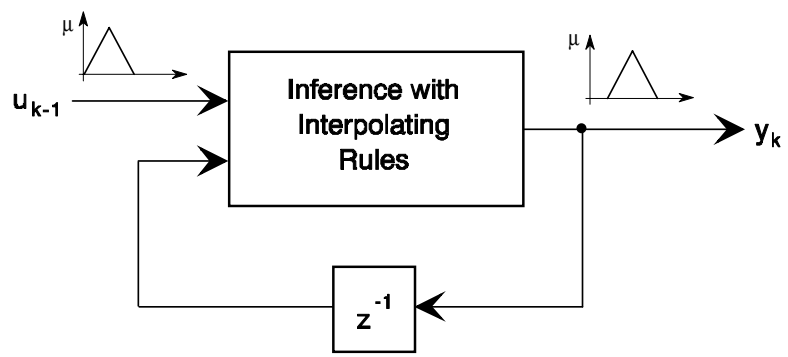

Figure 3. Dynamic Fuzzy System
To show the basic behavior of such a fuzzy system and to come to an appropriate stability definition, it is sufficient to look at a simple undriven fuzzy system only described by the following two rules

$$
\begin{aligned}
& \text { IF } Y_{k-1} \text { is "negative" THEN } Y_{k} \text { is "positive" } \\
& \text { IF } Y_{k-1} \text { is "positive" THEN } Y_{k} \text { is "negative". }
\end{aligned}
$$

The interpolation domain spanned by the two linguistic values " $Y_{k-1}$ is positive" and " $Y_{k-1}$ is negative" is the universe of discourse of the linguistic variable $Y_{k-1}$. The membership functions defined on the input domain are shown in figure 4. Depending on the output membership functions, the system shows different dynamic behavior. Given the output membership functions of figure 5, we obtain system 1 which is stable since the fuzzy output converges to the fuzzy number $\mathrm{Y}_{\infty}=\langle 0 ;-2,2\rangle$ for any initial fuzzy state. Figure 6 depicts the fuzzy output resulting from a crisp initial state $\mathrm{Y}_{0}=\langle 2 ; 2,2\rangle$.

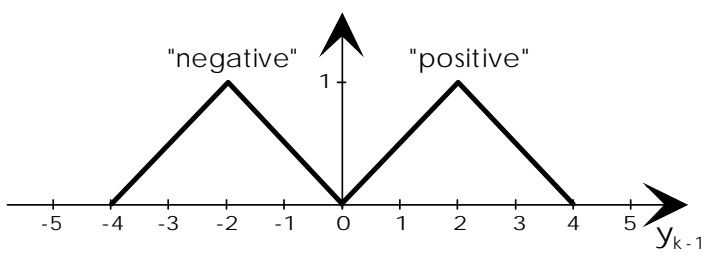

Figure 4. Membership functions of the input domain

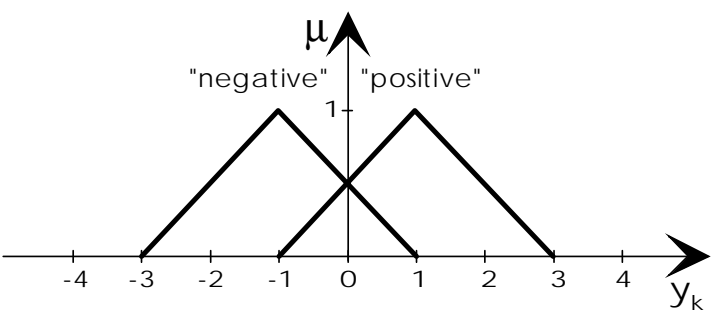

Figure 5. Output domain membership functions

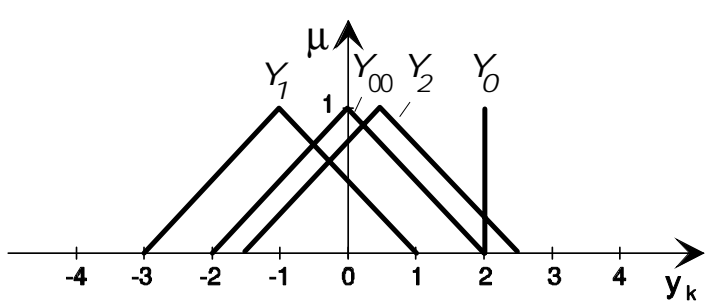

Figure 6. Simulation of the fuzzy system 1 
The output membership functions of system 2 shown in figure 7 cause an unstable system behavior. Although the center of the output converges to 0 for any initial state, its left and right foot move to infinity (fig. 8). Since the output is getting fuzzier with every step, the specificity of the output vanishes for $\mathrm{k} \rightarrow \infty$.

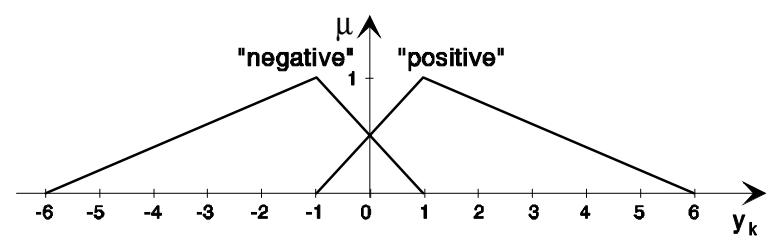

Figure 7. Too fuzzy output membership functions cause an unstable system behavior for system 2

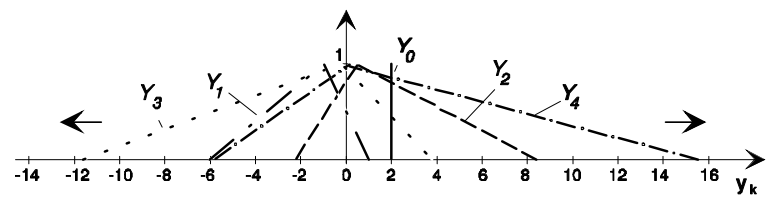

Figure 8. Specificity of the fuzzy output decreases with each step

These simple examples suggest the following stability definition for dynamic fuzzy systems:

An equilibrium point of a fuzzy system marked by a crisp value $R_{0}$ is (asymptotically) stable iff

- $\mathrm{R}_{0}$ is an (asymptotically) stable equilibrium point for the center of the output $y_{k}$

- the feet of the fuzzy output stay in a bounded environment of $\mathrm{R}_{0}$.

The equilibrium point of the two examples above is marked by $R_{0}=0$. System 1 has one asymptotically stable equilibrium point, whereas the equilibrium point of system 2 is unstable.

Since it is sufficient to examine the mapping of the crisp parameters of the fuzzy input onto the crisp parameters of the fuzzy output, usual methods for the stability analysis of nonlinear systems can be applied. If the membership functions defined on the input domain $\mathrm{y}_{\mathrm{k}-1}, \ldots \mathrm{y}_{\mathrm{k}-\mathrm{n}}$ are fuzzier than the membership functions defined on the output domain $y_{k}$, it is only necessary to analyze the mapping of the centers of the fuzzy input onto the fuzzy output. With a constant fuzzy $U_{k}$ it results a discrete nonlinear system described by

$$
\mathrm{y}_{\mathrm{k}, \mathrm{c}}=\mathrm{f}\left(\mathrm{y}_{\mathrm{k}-1, \mathrm{c}}, \ldots \mathrm{y}_{\mathrm{k}-\mathrm{n}, \mathrm{c}}\right)
$$

with the centers $\mathrm{y}_{\mathrm{k}, \mathrm{c}}, \mathrm{y}_{\mathrm{k}-1, \mathrm{c}}, \ldots \mathrm{y}_{\mathrm{k}-\mathrm{n}, \mathrm{c}}$ of the fuzzy output $Y_{k}$ and its delays $Y_{k-1}, \ldots Y_{k-n}$. To analyze such a system, methods based on common stability analysis approaches may be used. The "Convex Decomposition" $[3,5]$ as an efficient numerical stability analysis method has been successfully applied to dynamic fuzzy systems. Considering first order dynamic fuzzy systems, the stability region of an equilibrium point might even be analytically determined.

\section{Fuzzy Controller Design based on Fuzzy Models}

In this chapter a method is presented to derive a fuzzy controller from a fuzzy set of rules describing the dynamic system behavior. The fuzzy controller is designed to stabilize the system in the desired equilibrium point.

A first order fuzzy system is used to explain the essential design steps. The underlying set of rules is

$$
\begin{array}{llllll}
\text { IF } & \mathrm{Y}_{\mathrm{k}-1}=A & \text { AND } & \mathrm{U}_{\mathrm{k}-1}=X & \text { THEN } & \mathrm{Y}_{\mathrm{k}}=A X \\
\text { IF } & \mathrm{Y}_{\mathrm{k}-1}=B & \text { AND } & \mathrm{U}_{\mathrm{k}-1}=X & \text { THEN } & \mathrm{Y}_{\mathrm{k}}=B X \\
\text { IF } & \mathrm{Y}_{\mathrm{k}-1}=C & \text { AND } & \mathrm{U}_{\mathrm{k}-1}=X & \text { THEN } & \mathrm{Y}_{\mathrm{k}}=C X \\
\text { IF } & \mathrm{Y}_{\mathrm{k}-1}=A & \text { AND } & \mathrm{U}_{\mathrm{k}-1}=Z & \text { THEN } & \mathrm{Y}_{\mathrm{k}}=A Z \\
\text { IF } & \mathrm{Y}_{\mathrm{k}-1}=B & \text { AND } & \mathrm{U}_{\mathrm{k}-1}=Z & \text { THEN } & \mathrm{Y}_{\mathrm{k}}=B Z \\
\text { IF } & \mathrm{Y}_{\mathrm{k}-1}=C & \text { AND } & \mathrm{U}_{\mathrm{k}-1}=Z & \text { THEN } & \mathrm{Y}_{\mathrm{k}}=C Z \text {. }
\end{array}
$$

The premises $A, B$ and $C$ are defined on the universe of discourse of the delayed output $\mathrm{y}_{\mathrm{k}-1}$ and the premises $X$ and $Z$ are defined on the universe of $\mathrm{u}_{\mathrm{k}-1}$. The conclusions defined on $\mathrm{y}_{\mathrm{k}}$ are not fuzzier than one of the premises $A, B$ or $C$. Therefore, it is sufficient to consider the mapping of the centers $y_{k-1, c}$ and $u_{k-1, c}$ onto the center $y_{k, c}$. Using the inference with interpolating rules to evaluate the fuzzy rule set, it can be shown [7] that

$$
\mathrm{y}_{\mathrm{k}, \mathrm{c}}=\mathrm{r}\left(\mathrm{y}_{\mathrm{k}-1, \mathrm{c}}\right)+\mathrm{h}\left(\mathrm{y}_{\mathrm{k}-1, \mathrm{c}}\right) \cdot \mathrm{u}_{\mathrm{k}-1, \mathrm{c}}
$$

with the continous functions $\mathrm{r}(\cdot)$ and $\mathrm{h}(\cdot)$ holds. Assuming $\mathrm{h}\left(\mathrm{y}_{\mathrm{k}-1, \mathrm{c}}\right) \neq 0 \forall \mathrm{y}_{\mathrm{k}-1, \mathrm{c}}$, the control law 


$$
\mathrm{u}_{\mathrm{k}-1, \mathrm{c}}=\frac{\mathrm{y}_{\mathrm{R}}-\mathrm{r}\left(\mathrm{y}_{\mathrm{k}-1, \mathrm{c}}\right)}{\mathrm{h}\left(\mathrm{y}_{\mathrm{k}-1, \mathrm{c}}\right)}
$$

makes sure that the center of the output $\mathrm{y}_{\mathrm{k}, \mathrm{c}}$ reaches the desired equilibrium point $y_{R}$ within a single step. Without limitation of the control signal, the stability region equals the domain of definition. However, in practical applications a limitation of the control signal has to be taken into account. Now, a stability region of the equilibrium point might be determined using a Ljapunov function, e.g.

$$
\mathrm{V}\left(\mathrm{y}_{\mathrm{k}, \mathrm{c}}\right)=\left(\mathrm{y}_{\mathrm{k}, \mathrm{c}}-\mathrm{y}_{\mathrm{R}}\right)^{2} \text {. }
$$

Thus, the first step of the controller design is to form the control law (1). Next, the stability region of the desired equilibrium point is determined under consideration of the limitation of the control signal. With (1), an adequate manipulation variable might be calculated for each center $y_{k-1, c}$ of the ascertained stability region. We are starting with the fuzzy controller rule set

$$
\begin{array}{llll}
\text { IF } & \mathrm{Y}_{\mathrm{k}-1}=A & \text { THEN } & \mathrm{U}_{\mathrm{k}-1}=\mathrm{u}_{\mathrm{A}} \\
\text { IF } & \mathrm{Y}_{\mathrm{k}-1}=B & \text { THEN } & \mathrm{U}_{\mathrm{k}-1}=\mathrm{u}_{\mathrm{B}} \\
\text { IF } & \mathrm{Y}_{\mathrm{k}-1}=C & \text { THEN } & \mathrm{U}_{\mathrm{k}-1}=\mathrm{u}_{\mathrm{C}} .
\end{array}
$$

The premises $A, B$ and $C$ are known from the rule set representing the process behavior, whereas the crisp conclusions $\mathrm{u}_{\mathrm{A}}, \mathrm{u}_{\mathrm{B}}$ and $\mathrm{u}_{\mathrm{C}}$ are calculated using (2). Thus, the conclusion of the first rule is given by

$$
\mathrm{u}_{\mathrm{A}}=\frac{\mathrm{y}_{\mathrm{R}}-\mathrm{r}\left(\mathrm{a}_{\mathrm{c}}\right)}{\mathrm{h}\left(\mathrm{a}_{\mathrm{c}}\right)} \text {. }
$$

Due to the singletons used as conclusions, crisp controller inputs lead to a crisp control output. Only for the crisp inputs $a_{c}, b_{c}$ and $c_{c}$ the evaluation of the controller rule set (3) using the inference with interpolating rules yields the same output as the crisp control law (2). If $\mathrm{y}_{\mathrm{k}-1, \mathrm{c}}$ is somewhere between these particular values, the controller output is determined by interpolation. It might be necessary to add more rules if the characteristics of the fuzzy controller differs too much from the nonlinear characteristics of control law (2).

For a dynamic fuzzy system of higher order as a process model equation

$$
u_{k-1, c}=\frac{y_{R}-r\left(y_{k-1, c}, \ldots y_{k-n, c}, u_{k-2, c}, \ldots u_{k-m, c}\right)}{h\left(y_{k-1, c}, \ldots y_{k-n, c}, u_{k-2, c}, \ldots u_{k-m, c}\right)}
$$

instead of equation (2) might be used as a basis for the controller design.

With the inference with interpolating rules, the fuzzy rule set resulting so far could be used for a fuzzy controller. Due to the crisp conclusions, a defuzzification would not be necessary for crisp inputs of the controller. Such a controller has a characteristics that consists of regions where multilinear functions are defined [7]. However, we get a controller with the same characteristics if the fuzzy rule set is evaluated with the common sum-prod-inference together with a center of singletons defuzzification. Only the premise membership functions have to be manipulated in the following way: The centers of all premises are kept but the feet are moved to the centers of the adjacent premises.

Finally, a fuzzy rule set with triangular membership functions for the premises and singletons for the conclusions results. This set of rules is used for a fuzzy controller which can be evaluated with well-known methods. Thus, the final tuning of the controller in the closed loop with the real process might be accomplished with common software tools.

\section{Control of a Continous Stirred Tank Reactor}

In the following, the new fuzzy controller design approach is applied to develop a fuzzy controller for a continous stirred tank reactor (fig. 9)[2].

To disintegrate to other substances a substance with the concentration $\mathrm{c}_{0}$ is dumped into a tank reactor with a catalyst inside. The temperature $\mathrm{T}$ in the tank is to be controlled on a setpoint in order that the incoming substance reacts with high intensity. The controller output is the coolant temperature $T_{k}$ which is $T_{0}$ in the setpoint.
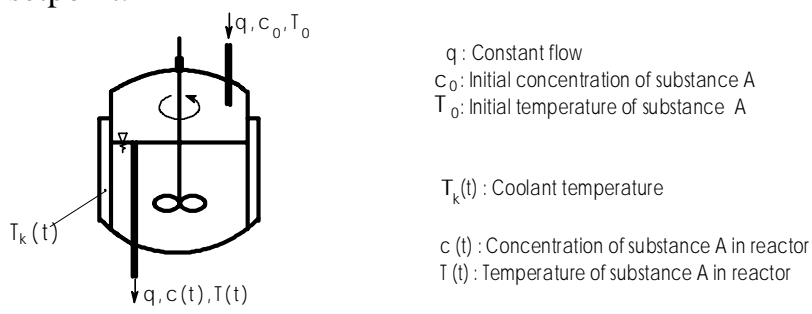

Figure 9. Continous stirred tank reactor 
As a simplification, we define $\mathrm{u}$ as the specific coolant temperature and $\tau$ as the specific temperature of the substance as follows:

$$
\mathrm{u}=\frac{\mathrm{T}_{\mathrm{k}}-\mathrm{T}_{0}}{\mathrm{~T}_{0}}, \quad \tau=\frac{\mathrm{T}-\mathrm{T}_{0}}{\mathrm{~T}_{0}} .
$$

In the desired setpoint the specific coolant temperature is zero and the specific temperature of the substance is 0,05 .

The fuzzy variable $\tau_{\mathrm{k}}$ defined on $\tau_{\mathrm{k}}$ is the fuzzy output of the linguistic process model. It depends on its delays $\tau_{\mathrm{k}-1}, \tau_{\mathrm{k}-2}$ and the delayed fuzzy inputs $\mathrm{U}_{\mathrm{k}-1}$ und $\mathrm{U}_{\mathrm{k}-2} .48$ rules like

IF $\mathrm{U}_{\mathrm{k}-2}$ neg AND $\mathrm{U}_{\mathrm{k}-1}$ pos AND $\tau_{\mathrm{k}-2}$ medium AND $\tau_{\mathrm{k}-1}$ big THEN $\tau_{\mathrm{k}}$ very big

characterize the process behavior. Based on this linguistic process model, a fuzzy controller with 24 rules is developed following the design steps of chapter 4. The behavior of the controlled process is examined in a simulation with an analytical nonlinear continous process model [2]. Figure 10 shows the controlled system in case of an initial temperature of the substance of $\tau_{R_{0}}=0,01$. The performance of the specific concentration of the substance

$$
\kappa=\frac{\mathrm{c}_{0}-\mathrm{c}}{\mathrm{c}_{0}}
$$

which can not be measured on the real process is also depicted. Even with a limited control signal of $|\mathrm{u}| \leq 0,04$ the controlled system shows a satisfactory behavior.
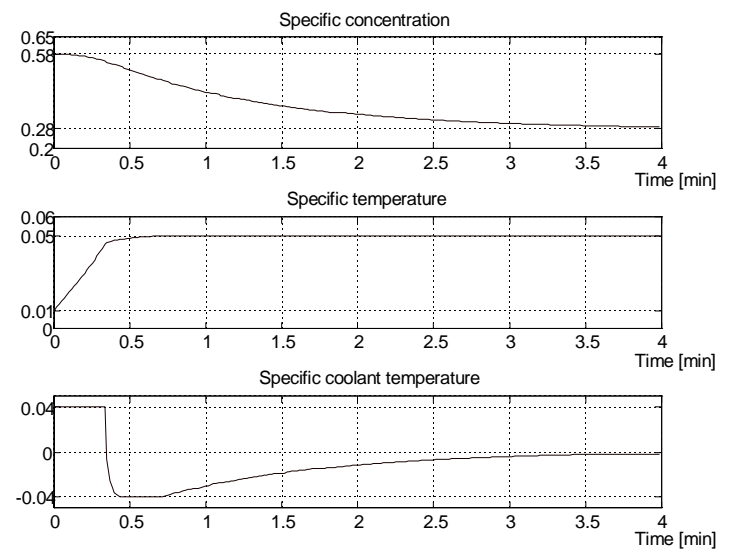

Figure 10. Behavior of the controlled stirred tank reactor
Thus, it may be concluded that the presented design method for fuzzy controllers is a powerful approach if only qualitative (linguistic) knowledge about the process is available.

\section{References}

[1] H. Bandemer, S. Gottwald: Einführung in FuzzyMethoden. Akademie Verlag, Berlin 1993.

[2] O. Föllinger: Nichtlineare Regelungen I, Oldenbourg Verlag, 1993

[3] H. Kiendl: Robustheitsanalyse von Regelungssystemen mit der Methode der konvexen Zerlegung. Automatisierungstechnik 35, pp. 192-202, 1987 (5).

[4] J. P. LaSalle: The Stability and Control of Discrete Processes. Applied Mathematical Sciences 62, SpringerVerlag, 1986.

[5] O. Rumpf: Anwendung der Methode der konvexen Zerlegung zur Stabilitätsanalyse dynamischer Systeme mit neuronalen Komponenten. Automatisierungstechnik 44, pp 101-107, 1996 (3).

[6] R. R. Yager, D. P. Filev: Essentials of Fuzzy Modeling and Control. John Wiley \& Sons, Inc. 1994.

[7] E. Schäfers, V. Krebs, M. Sackmann: Dynamische Fuzzy-Systeme: Stabilitätsanalyse und Reglerentwurf. 6 . Workshop "Fuzzy-Control" des GMA-UA 1.4.2, 1996. 